\title{
Role of Magnetic Resonance Imaging in evaluation of postoperative knee surgery
}

\author{
Mahmoud Mohamed Ahmed Ali ${ }^{1}$, Mohamed Ali Aboud ${ }^{1}$, Mohamed Salah El-Feshawy ${ }^{1}$, \\ Ali Mohamed El-Gyoshy ${ }^{2}$
}

\author{
Department of ${ }^{1}$ Radiodiagnosis and ${ }^{2}$ Orthopedic Surgery, Faculty of medicine - Al-Azhar University \\ Corresponding author: Mahmoud Mohamed Ahmed Ali, E-Mail: mahmouddr75@gmail.com, Mobile: -01020881293
}

\begin{abstract}
Background: MRI has high sensitivity and specificity regarding the assessment of the post-operative knee joint. Purpose: to assess the role of MRI in evaluation of the post-operative knee joint and assessment of the post complications after ACL, meniscal and cartilage surgical repair procedures. Patients and Methods: this study included 20 patients, their age range between 14-50 years, all presented postoperative knee were referred to Radiology Department at El-Hussien University Hospital and private centers for MRI exam for postoperative assessment, complication or follow up after orthopedic examination from Orthopedic Department at El-Hussien Hospitals and Outpatient Clinic. Patients had MRI imaging of the affected knee joint using high field strength scanners (1.5 T) MRI unit (Achieva, Philips medical system) MRI was performed by Knee coil in all cases. Results: this study included 15(75\%) males and 5 females (5\%)(A) Due to different types of operations $10(50 \%)$ had ACL reconstruction, 6(30\%) had menisectomy,4(20\%) had cartilage repair. (B) Due to causes $16(80 \%)$ trumatic,4(20\%)non trumatic. (C) Due to types of complications: $3(15 \%)$ had no complications, $12(60 \%)$ had swelling, 2(10\%) had limitation of movement,2(10\%) had pain and limitation, 1(5\%)had pain and swelling. Conclusion: MRI proved as an accurate method for evaluation of the knee joint after ACL, meniscus and cartilage repair that can help predict post-operative complications.
\end{abstract}

Keywords: MRI postoperative knee, Postoperative knee complications.

\section{INTRODUCTION}

The knee is a frequently injured joint and, thus, a common focus of operative intervention. As operative techniques and imaging modalities evolve, radiologist must be aware of the expected postoperative appearance after knee surgeries that are performed commonly, and also must be comfortable recognizing complications encountered in the immediate or delayed postoperative period ${ }^{(\mathbf{1})}$.

Magnetic resonance imaging (MRI) is often the method of choice to evaluate soft tissue injuries in acute knee trauma, and has widely replaced diagnostic arthroscopy. MRI is a noninvasive examination that allows excellent soft tissue contrast without ionizing radiation ${ }^{(2)}$.

MRI of the knee after surgical repair is becoming more common because of the increasing number of therapeutic knee arthroscopic procedures being performed. It is important to understand the surgical procedure performed as well as the normal postoperative MR appearance to make it possible to diagnose complications following such procedures ${ }^{(3)}$.

The most common arthroscopic repair procedures include partial meniscectomy and meniscal repair, anterior cruciate ligament (ACL) reconstruction, and cartilage repair procedures ${ }^{(3)}$.

Meniscal tears are the most frequent injury to the knee joint. Such injuries are especially prevalent among competitive athletes. In the last 25 years, the number of people participating in sports has greatly increased, resulting in a higher number of knee injuries ${ }^{(4)}$.

Anterior cruciate ligament is considered the most common ligament that can be reconstructed. Clinical evaluation of ACL reconstructions can be difficult, and MRI plays an important role in evaluating the integrity of the ACL graft, as well as diagnosis of complications associated with ACL reconstruction ${ }^{(5)}$.

Posterior cruciate ligament (PCL) is considered the second ligament that exposes to injuries. The accuracy of MRI in the evaluation of PCL injuries (near 100\% sensitivity and specificity) has been described ${ }^{(\boldsymbol{G})}$.

Articular cartilage injury is very common, being present in up to $63 \%$ of arthroscopic procedures. With the advent of new cartilage repair procedures that have the potential to either form hyaline-like repair tissue or transplant hyaline cartilage to the damaged area, there is an increased need for an accurate noninvasive method to evaluate the results of such repair procedures. MR imaging is currently the best method for such evaluation ${ }^{(7)}$.

\section{AIM OF THE WORK}

The aim of our study is to find out the role of MRI in evaluation of postoperative knee.

\section{PATIENT AND METHODS}

This study included 20 patients, their age range between 14-50 years, all presented postoperative knee 
were referred to Radiology department at El-Hussien University Hospital and private centers for MRI exam for post-operative assessment, complication or follow up after orthopedic examination from Orthopedic Department at El-Hussien Hospitals and Outpatient Clinic. The study was approved by the Ethics Board of Al-Azhar University.

Patients had MRI imaging of the affected knee joint using high field strength scanners $(1.5 \mathrm{~T})$ MRI unit (Achieva, Philips medical system) MRI was performed by Knee coil in all cases.

Inclusion criteria: Post-operative Patient of both sex. Any patient with a history of knee joint intervention with complication.

Exclusion Criteria: Early pregnant patient. Knee joint tumor. Any electrically, magnetically or mechanically activated implant, cardiac pace maker, cochlear implant and hearing aids. Chronic muscle disorders. Known active articular infection. Metabolic bone disease \&neoplastic disease.

MRI protocol: Axial T2, Coronal STIR, Sagittal T1, Sagittal T2, Sagittal STIR as table shows below:

Table (1): MRI sequences parameter on high field strength scanners.

\begin{tabular}{|c|c|c|c|c|c|c|}
\hline Parameter & $\begin{array}{c}\text { Sagittal } \\
\text { T1 }\end{array}$ & $\begin{array}{c}\text { Sagittal } \\
\text { T2 }\end{array}$ & $\begin{array}{c}\text { Sagittal } \\
\text { STIR }\end{array}$ & $\begin{array}{c}\text { Sagittal } \\
\text { PD }\end{array}$ & $\begin{array}{c}\text { Coronal } \\
\text { T2 FSE }\end{array}$ & $\begin{array}{c}\text { Axial } \\
\text { T2 }\end{array}$ \\
\hline TE & 17 & 100 & 60 & 30 & 13 & 100 \\
\hline $\begin{array}{c}\text { FOV } \\
\text { Ant./post }\end{array}$ & 30 & 30 & 30 & 30 & 30 & 20 \\
\hline
\end{tabular}

Statistical analysis of MRI images: The assessment of the injury concerned is based on the signal intensity, location and morphology of region of concern in all sequences in correlation with clinical data, clinical examination and previous exams.

\section{RESULTS}

This study included $15(75 \%)$ males and 5 females (5\%)as table shows below:

Table (2): Shows General characteristics of the study population.

\begin{tabular}{|l|l|c|c|}
\hline \multicolumn{2}{|c|}{} & Frequency & Percent \\
\hline \multirow{3}{*}{ Age } & Minimum & Maximum & Mean \pm SD \\
\cline { 2 - 4 } & 14 & 50 & $32.25 \pm 13.533$ \\
\hline \multirow{2}{*}{ Sex } & Female & 5 & 25.0 \\
\cline { 2 - 4 } & Male & 15 & 75.0 \\
\hline
\end{tabular}

In this study results due to different types of operations 10/20 (50\%) had ACL reconstruction, $6 / 20$ (30\%) had menisectomy, 4/20 (20\%) had cartilage repair as table shows below:

Table (3): Shows Distribution of different types of operations.

\begin{tabular}{|l|l|c|c|}
\hline \multicolumn{2}{|c|}{} & Frequency & Percent \\
\hline \multirow{2}{*}{ ACL } & No & 10 & $50.0 \%$ \\
\cline { 2 - 4 } & Yes & 10 & $50.0 \%$ \\
\hline \multirow{2}{*}{ Meniscus } & No & 14 & $70.0 \%$ \\
\cline { 2 - 4 } & Yes & 6 & $30.0 \%$ \\
\hline Cartilage & No & 16 & $80.0 \%$ \\
\hline Repair & Yes & 4 & $20.0 \%$ \\
\hline
\end{tabular}

In this study results due to causes included $16 / 20(80 \%)$ trumatic,4/20(20\%)non trumatic and due to types of complications:3/20(15\%)had no complications, 12/20 (60\%) had swelling, 2/20 (10\%) had limitation of movement, $2 / 20(10 \%)$ had pain and limitation, 1/20(5\%)had pain and swelling as table shows below:

Table (4): Shows Distribution of the cause and types of complications.

\begin{tabular}{|c|c|c|c|}
\hline & & Frequency & Percent \\
\hline \multirow{4}{*}{ Cause } & Non traumatic & 4 & $20.0 \%$ \\
\hline & Traumatic & 16 & $80.0 \%$ \\
\hline & Nothing & 3 & $15.0 \%$ \\
\hline & Swelling & 12 & $60.0 \%$ \\
\hline \multirow{3}{*}{ Complication } & $\begin{array}{c}\text { Limitation of } \\
\text { movement }\end{array}$ & 2 & $10.0 \%$ \\
\hline & Pain \& limitation & 2 & $10.0 \%$ \\
\hline & Pain \& swelling & 1 & $5.0 \%$ \\
\hline Postoperative & Minimum & Maximum & Mean \pm Standard \\
\hline \multirow{2}{*}{ Duration } & & & Deviation \\
\hline & 2 & 84 & $16.90+16.827$ \\
\hline A & & B & 5 \\
\hline
\end{tabular}

Figure (1): 41 year old male patient complaining of knee pain 1 year following ACL reconstruction (A and B) Proton density (PD) fat suppressed sagittal and coronal images showing focal cartilage defect at the lateral femoral condyle. 

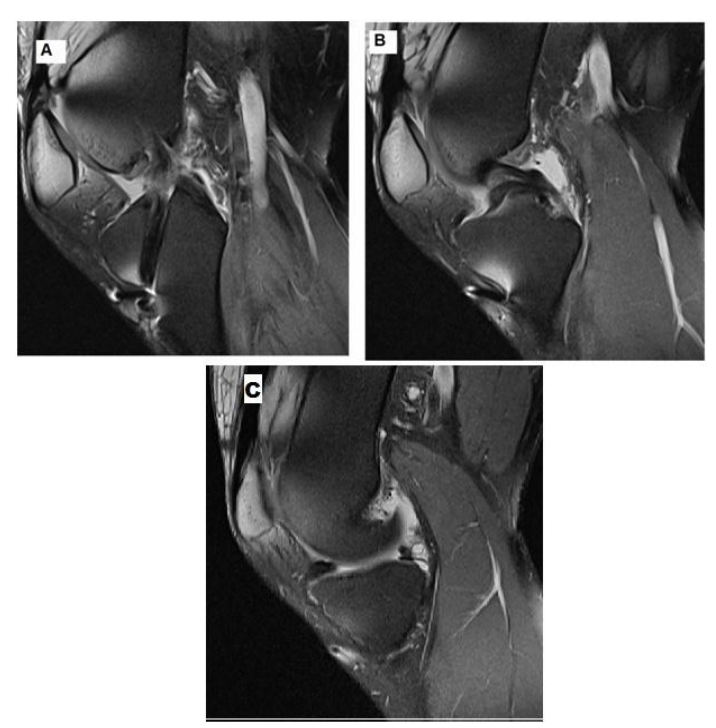

Figure (2): Male Patient 25 years 10 months post ACL surgery with history of recent trauma and inability to walk.(A), (B) and (C) sagittal oblique PD fat saturation MRI demonstrate tear of the ACL graft at the tibial margin and Bucket handle tear of posterior horn of the medial meniscus.

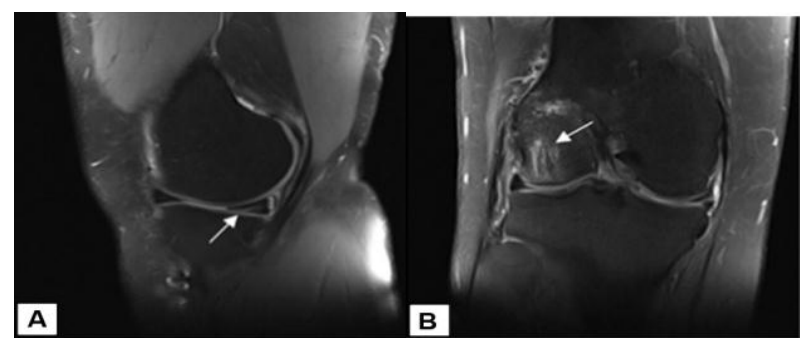

Figure (3): 33 year old male complaining of pain and giving way 2 years after ACL reconstruction.(A) and(B) Sagittal and coronal PD fat suppressed images revealing torn posterior horn of the medial meniscus and bone contusion of the lateral femoral condyle.
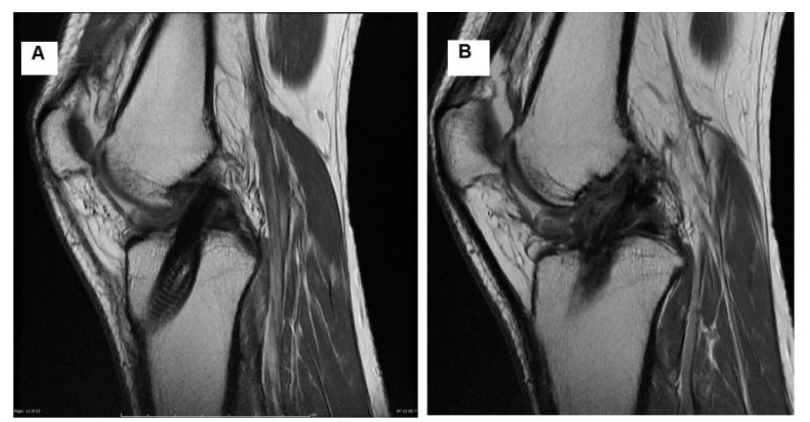

Figure (4): Male Patient 20 years complains Knee pain after 2 years of ACL reconstruction.(A) and (B) Sagittal PD MRI images show intact ACL graft with secondary ligamentization of the graft and prominent scarring anterior to the ACL graft projecting into the inferior aspect of Hoffa's fat pad compatible with cyclop lesion (also known as localized anterior arthrofibrosis).

\section{DISCUSSION}

The most commonly reconstructed ligament in the knee is the ACL. Its clinical evaluation can be difficult. Post-operative ACL graft patients complaining of knee instability and loss of extension, limitation of movement, swelling, or pain are indicated for clinical and radiological examination aiming to diagnose ACL graft failure, ACL graft complication or other internal derangement ${ }^{(\mathbf{1 2})}$.

In our study 2 patients (20\%) had graft impingement which was the commonest post-operative complication, the tibial tunnel was seen anterior to the intersection of the slope of the intercondylar roof with the proximal tibia in these patients where the graft was seen impinged on by the roof of the inter-condylar notch as the tibial tunnel should be oriented parallel to the Blumensaat line which is a line drawn along the intercondylarro of, its distal portion should start near the tibial tuberosity, and the intra articular opening of the tunnel should be completely posterior to this line. In some of these patients not only the tibial tunnel was malpositioned also the femoral tunnel was also seen malpositioned. This was concordant with ${ }^{(12)}$.

The patient population in our study for the ACL repair had the following associated MRI findings: 8 patients had posterior hom medial meniscus (PHMM) tear, 2 patients had posterior horn lateral meniscus (PHLM) tear, 6 patients had joint effusion, 2 patients had diffuse arthrofibrosis, 1 patient had medial collateral ligament sprain, 1 patient had anterior tibial translation, 1 patients had uncovered PHLM, and 1 patient had Cyclops lesion.It is known that MRI is a non-invasive method to assess meniscal status and therefore easily accepted by patients. However, the evaluation of the meniscus after resection or repair is difficult to evaluate with MRI. Many authors proposed different, and not the classic MR criteria to differentiate repeat meniscal tears after meniscal resection ${ }^{(0)}$.

In our study a repairedmeniscus was considered healed if there was neither joint line tenderness, nor effusion or a positive McMurrey test, according to the strict clinical criteria of ${ }^{(\mathbf{1 5}) \text {. }}$

According to the literature the appearance of the meniscus after meniscal repair has a grade three signal intensity on MRI scans postoperatively in the majority of cases in the healing meniscus suggested that the presence of grade three signal intensity within the postoperative meniscus was not an indicator of recurrent meniscal tear ${ }^{(\mathbf{1 3 )}}$.

in an excellent study found that during the normal healing of a repaired meniscus there is a gap of $1-2 \mathrm{~mm}$ between the repaired segments, which filled 
with a translucent tissue highly cellular and fibrovascular 3 months after repair. Six months postoperatively, the gap is gradually filled with the repair tissue showing evidence of fibrocartilagenous metaplasia, gradual filling of the gap, which is part of the normal course of meniscal healing, can be evaluated with conventional MRI. Our findings were in agreement with the above mentioned publications ${ }^{(\mathbf{1 4})}$.

Retorn meniscus was found in 1 of our patients (16.66\%), the findings of abnormal meniscal morphology, high-signal-intensity joint fluid extending into a cleft within the meniscal fragmention T2-weighted images or reaching to articular surface as well as a displaced meniscal fragment are specific and but less sensitive signs of a retorn meniscus, this is in agreement with who stated that using of the stricter criterion of fluid signal intensity within a linear defect in the meniscus on T2-weighted images has been shown to provide high specificity $(88 \%-92 \%)$ but low sensitivity $(41 \%-69 \%)$ for tears ${ }^{(16)}$.

Joint effusion was observed in our study in 1 patients $(16,66 \%)$ and was associated with an overall incremental increase in accuracy in the diagnosis of a recurrent meniscal tear, in accordance with ${ }^{(\mathbf{1 7 )}}$.

\section{CONCLUSIONS}

In conclusion our findings proved that MRI has high sensitivity and specificity regarding the assessment of the post-operative knee joint also all the above mentioned papers stressed that MRI imaging provides excellent anatomical and morphological assessment of the knee joint after surgical intervention in the field of ACL, meniscal and cartilage repair being time saving and non-invasive as well as being available on a broad spectrum.

\section{REFERENCES}

1. Frick MA, Collins MS, Adkins MC (2006): MR Imaging of the Postoperative Knee. Radiologic Clinics of North America, 344: 367-389.

2. Vande Berg BC, Lecouvet FE, Poilvache $P$ et al. (2009): Anterior cruciate ligament tears and associated meniscal lesions assessment at dualdetector spiral CT arthrography. Radiology, 223:403-409.

3. Recht MP, Kramer J (2010): MR Imaging of the Postoperative Knee. The Department of Medical Imaging, Mount Sinai Hospital and the University Health Network, University of Toronto.

4. Johnson MJ, Lucas GL, Dusek JK et al. (2009): Isolated Arthroscopic Meniscal repair: a LongTerm Outcome Study (More Than 10 Years). American Journal of Sports Medicine, 27:44-49.
5. Wada $Y$, Watanabe $A$, Yamashita $T$ et al. (2010): Evaluation of Articular Cartilage with 3D MR after Autologous Chondrocyte Implantation. Journal of Orthopedic Science, 8:514- 517.

6. Shelbourne KD, Jennings RW, Vahey TN (2012): Magnetic Resonance Imaging of Posterior Cruciate Ligament Injuries, American Journal of Knee Surgery, 12:209-213.

7. Sirlin CB, Brossman J, Boutin RD et al. (2010): Osteochondral Allografts of the Knee: Comparison of MR Imaging Findings and Immunologic Responses. Radiology, 219:35-43.

8. DT, Felson (2012): An Update on the Patheogenesis and Epidemiology of Osteoarthritis. Radiology Clinic North America, 1:1-9.

9. May DA, Snearly WN, Bents R, Jones R (2009): MR Imaging Findings in Anterior Cruciate Ligament Reconstruction: Evaluation of Notchplasty, 9:217-221.

10. Marlovits $S$, Zeller $P$, Singer $P$ et al. (2006): Cartilage Repair Generations of Autologous Chondrocyte Transplantation. European Journal of Radiology, 57:24-31.

11. Meyers AB (2010): Imaging of anterior cruciate ligament repair and its complications. AJR Am J Roentgenol., 194:476-84.

12. Crema MD, Roemer FW, Marra MD et al. (2011): Articular cartilage in the knee: current MR imaging techniques and applications in clinical practice and research. Radiographics,31 (1):37-61.

13. Farley TE, Howell SM, Love KF et al. (2011): Meniscaltears: MR and arthrographic findings after arthroscopic repair. Radiology, 180:517-22.

14. Arnoczky SP, Cooper TG, Stadelmaier DM et al. (2004): Magnetic resonance signals in healing menisci. Arthroscopy,10:552-7.

15. Barrett GR, Field MH, Treacy SH et al. (2008): Clinical results of meniscusrepair in patients 40 years and older. Arthroscopy, 14:824-9.

16. Chung CB, Frank LR, Resnick D(2011): Cartilage imaging techniques: current clinical applications and state of the art imaging. Clinical Orthopedic Related Resonance, 391(1): 370-378.

17. Wood JJ, Malek MA, Frassica FJ et al. (2006): Autologous Chondrocyte Transplantation: Adverse Events Reported to the United States FDA. Bone Joint Surgery Journal of America, 88:503-507.

18. Brown WE, Potter HG, Marx RG et al. (2004): Magnetic Resonance Imaging Apperance of Cartilage Repair in the Knee. Clinical Orthopedic Related Resonance, 422:214-223. 